\title{
Numerical Solution of Nonlinear Diffusion Equation with Convection Term by Homotopy Perturbation Method
}

\author{
Saad A. Manaa ${ }^{1}$,Fadhil H. Easif ${ }^{2}$, Bewar A. Mahmood ${ }^{3}$ \\ ${ }^{1,2,3}$ Department of Mathematics, Faculty of Science, University of Zakho,Duhok, Kurdistan Region, Iraq
}

\begin{abstract}
In this paper, an application of homotopy perturbation method (HPM) is applied to finding the approximate solution of nonlinear diffusion equation with convection term, We obtained the numerically solution and compared with the exact solution.The results reveal that the homotopy perturbation method is very effective, simple and very close to the exact solution.
\end{abstract}

Keywords: Diffusion equation with convection term, homotopy perturbation method.

\section{INTRODUCTION}

In recent years, the application of the homotopy perturbation method (HPM) $[1,2]$ in nonlinear problems has been developed by scientists and engineers, because this method continuously deforms the difficult problem under study into a simple problem which is easy to solve. The homotopy perturbation method [3], proposed first by He in 1998 and was further developed and improved by He [2, 4, 5]. The method yields a very rapid convergence of the solution series in the most cases. Usually, one iteration leads to high accuracy of the solution. Although goal of He's homotopy Perturbation method was to find a technique to unify linear and nonlinear, ordinary or partial differential equations for solving initial and boundary value problems. Most perturbation methods assume a small parameter exists, but most nonlinear problems have no small parameter at all. A review of recently developed nonlinear analysis methods can be found in [6]. Recently, the applications of homotopy perturbation theory among scientists were appeared [7-11], which has become a powerful mathematical tool, when it is successfully coupled with the perturbation theory $[2,5,12]$.

\section{I.1 Mathematical Model}

we consider the nonlinear diffusion equation with convection term of the form:

$u_{t}=\left(A(u) u_{x}\right)_{x}+B(u) u_{x}+C(u)$

Where $u=u(x, t)$ is the unknown function and $A(u), B(u)$, and $C(u)$ are arbitrary smooth functions on $u$. The indices $t$ and $x$ denotes differentiating with respect to the variables. The study of solution of this problem has over the years attracted the interest of many researches. Chemiha and Serov [13] considered the lie and non-lie symmetries of non linear diffusion with convection term. In this work, we will apply homotopy perturbation method to approximate solution of the generalized non linear diffusion equation with convection term of the form:

$u_{t}=a u_{x x}+b u u_{x}+c u(u-k)(u+k)$

with taking $A(u)=a, B(u)=b u$, and $C(u)=c u(u-k)(u+k)$

with the initial condition

$u(x, 0)=f(x), \quad \alpha \leq x \leq \beta$

\section{Materials And Methods}

II.1 Basic Idea of Homotopy Perturbation Method (HPM)

To illustrate HPM consider the following nonlinear differential equation:

$A(u)-f(r)=0, \quad r \in \Omega$,

with boundary conditions:

$B\left(u, \frac{\partial u}{\partial n}\right)=0, \quad r \in \Gamma$

Where $A$ is a general differential operator, $B$ is a boundary operator, $f(r)$ is a known analytic function and $\Gamma$ is the boundary of the domain $\Omega$.The operator $A$ can be generally divided into two parts $F$ and $N$, where $F$ is linear, Whereas $N$ is nonlinear. Therefore, equation (2) can be rewritten as follows:

$F(u)+N(u)-f(r)=0$

He [14] constructed a homotopy $v: \Omega \times[0,1] \rightarrow \mathrm{R}$ which satisfies:

$H(v, p)=(1-p)\left[F(v)-F\left(v_{0}\right)\right]+p[A(v)-f(r)]=0$,

$H(v, p)=F(v)-F\left(v_{0}\right)+p F\left(v_{0}\right)+p[N(v)-f(r)]=0$, 
Where $\mathrm{r} \in \Omega, p \in[0,1]$ that is called homotopy parameter, and $\mathrm{v}_{0}$ is an initial approximation of equation (2). Hence, it is obvious that:

$H(v, 0)=F(v)-F\left(v_{0}\right)=0, \quad H(v, 1)=A(v)-f(r)=0$,

and the changing process of $\mathrm{p}$ from 0 to 1 , is just that of $\mathrm{H}(\mathrm{v}, \mathrm{P})$ from $\mathrm{F}(\mathrm{v})-\mathrm{F}\left(\mathrm{v}_{0}\right)$ to $\mathrm{A}(\mathrm{v})-\mathrm{f}(\mathrm{r})$.

In topology, this is called deformation, $F(v)-F\left(v_{0}\right)$ and $A(v)-f(r)$ are called homotopic. Applying the perturbation technique [15], due to the fact that $0 \leq p \leq 1$ can be considered as a small parameter, we can assume that the solution of equation(5) equation (6) can be expressed as a series in $p$, as follows:

$v=v_{0}+p v_{1}+p^{2} v_{2}+p^{3} v_{3}+\cdots$,

when $p \rightarrow 1$, equation(5) or equation (6) corresponds to equation(4) and becomes the approximate solution of equation (4), i.e.,

$u=\lim _{p \rightarrow 1}(v)=v_{0}+v_{1}+v_{2}+v_{3}+\cdots$

The series (9) is convergent for most cases, and the rate of convergence depends on $A(v),[3]$.

\section{II.2 Solution of the nonlinear diffusion equation with convection term by HPM}

For solving equation (1), by homotopy perturbation method, we construct the following homotopy:

$H(v, p)=(1-p)\left[\frac{\partial v}{\partial t}-\frac{\partial u_{0}}{\partial t}\right]+p\left[\frac{\partial v}{\partial t}-a \frac{\partial^{2} v}{\partial x^{2}}-b v \frac{\partial v}{\partial x}-c v[(v-k)(v+k)]\right]=0$

hence, we get

$\frac{\partial v}{\partial t}-\frac{\partial u_{0}}{\partial t}-p \frac{\partial v}{\partial t}+p \frac{\partial u_{0}}{\partial t}+p \frac{\partial v}{\partial t}-p\left(a \frac{\partial^{2} v}{\partial x^{2}}\right)-p\left(b v \frac{\partial v}{\partial x}\right)-p[c v(v-k)(v+k)]=0$

Substituting equation (8) in equation (11), we get:

$\frac{\partial}{\partial t}\left(v_{0}+p v_{1}+p^{2} v_{2}+\cdots\right)-\frac{\partial u_{0}}{\partial t}-p \frac{\partial}{\partial t}\left(v_{0}+p v_{1}+p^{2} v_{2}+\cdots\right)$

$+p \frac{\partial u_{0}}{\partial t}+p \frac{\partial}{\partial t}\left(v_{0}+p v_{1}+p^{2} v_{2}+\cdots\right)-p a \frac{\partial^{2}}{\partial x^{2}}\left(v_{0}+p v_{1}+p^{2} v_{2}+\cdots\right)$

$-p b\left[\left(v_{0}+p v_{1}+p^{2} v_{2}+p^{3} v_{3}+\cdots\right)\left(\frac{\partial}{\partial x}\left(v_{0}+p v_{1}+p^{2} v_{2}+\cdots\right)\right)\right]$

$-p c\left[\begin{array}{c}\left(v_{0}+p v_{1}+p^{2} v_{2}+\cdots\right)\left(v_{0}+p v_{1}+p^{2} v_{2}+\cdots-k\right) \\ \left(v_{0}+p v_{1}+p^{2} v_{2}+\cdots+k\right)\end{array}\right]=0$

Collecting the terms with the same power of $p$, we get:

$$
\begin{array}{ll}
p^{0}: \frac{\partial v_{0}}{\partial t}-\frac{\partial u_{0}}{\partial t}=0 & , v_{o}(x, t)=u_{o}(x, t) \\
p^{1}: \frac{\partial v_{1}}{\partial t}+\frac{\partial u_{0}}{\partial t}-a \frac{\partial^{2} v_{0}}{\partial x^{2}}-b v_{0} \frac{\partial v_{0}}{\partial x}-c\left(v_{0}{ }^{3}-v_{0} k^{2}\right)=0 & , v_{1}(x, 0)=0 \\
p^{2}: \frac{\partial v_{2}}{\partial t}-a \frac{\partial^{2} v_{1}}{\partial x^{2}}-b\left(v_{0} \frac{\partial v_{1}}{\partial x}+v_{1} \frac{\partial v_{0}}{\partial x}\right)-c\left(3 v_{0}{ }^{2} v_{1}-v_{1} k^{2}\right)=0 & , v_{2}(x, 0)=0 \\
p^{3}: \frac{\partial v_{3}}{\partial t}-a \frac{\partial^{2} v_{2}}{\partial x^{2}}-b\left(v_{0} \frac{\partial v_{2}}{\partial x}+v_{1} \frac{\partial v_{1}}{\partial x}+v_{2} \frac{\partial v_{0}}{\partial x}\right)-c\left(3 v_{0}{ }^{2} v_{2}+3 v_{0} v_{1}{ }^{2}-v_{2} k^{2}\right)=0, v_{3}(x, t)=0
\end{array}
$$

$\vdots$

So we can calculate the equations $(13,14,15,16, \ldots)$ by applying the inverse operator on the interval $(0, t)$

$\mathrm{L}_{\mathrm{t}}^{-1}=\int_{0}^{\mathrm{t}}(.) \mathrm{dt}$,

From equation (13), we get

$v_{0}=u_{0}$

From equation (14), we get

$v_{1}(x, t)=\int_{0}^{t}\left(a \frac{\partial^{2} v_{0}}{\partial x^{2}}+b v_{0} \frac{\partial v_{0}}{\partial x}+c\left(v_{0}{ }^{3}-k^{2} v_{0}\right)-\frac{\partial u_{0}}{\partial t}\right) d t$ 


$$
\begin{aligned}
& =\int_{0}^{t} a \frac{\partial^{2} v_{0}}{\partial x^{2}} d t+\int_{0}^{t} b v_{0} \frac{\partial v_{0}}{\partial x} d t+\int_{0}^{t} c\left(v_{0}^{3}-k^{2} v_{0}\right) d t \\
& =a \frac{\partial^{2} v_{0}}{\partial x^{2}} t+b v_{0} \frac{\partial v_{0}}{\partial x} t+c\left(v_{0}^{3}-k^{2} v_{0}\right) t \\
& =\left(a \frac{\partial^{2} v_{0}}{\partial x^{2}}+b v_{0} \frac{\partial v_{0}}{\partial x}+c\left(v_{0}^{3}-k^{2} v_{0}\right)\right) t
\end{aligned}
$$

By the same way we can continue, Then the approximate solution of equation (1) is:

$$
\begin{aligned}
u(x, t)=\lim _{p \rightarrow 1} v & =\lim _{p \rightarrow 1}\left(v_{0}+v_{1} p^{1}+v_{2} p^{2}+v_{3} p^{3}+\cdots+v_{j} p^{j}+\cdots\right) \\
& =v_{0}+v_{1}+v_{2}+v_{3}+\cdots+v_{j}+\cdots
\end{aligned}
$$

\section{Nunerical Applications}

We will apply homotopy perturbation method (HPM) to solve the nonlinear diffusion equation with convection term, and present numerical results to verify the effectiveness of this method, we take the following example:

\section{III.1. Numerical Example and Results}

In this section, we present examples of nonlinear diffusion equation with convection term and results will be compared with the exact solutions.

\section{III.2. Example}

Consider the following nonlinear diffusion equation with convection term. [16]

$u_{t}=a u_{x x}+b u u_{x}+\frac{b^{2}}{9 a} u(u-k)(u+k)$

with the initial condition

$u(x, o)=\frac{k\left(-1+c_{1} e^{\frac{b k x}{3 a}}\right)}{1+c_{1} e^{\frac{b k x}{3 a}}+c_{2} e^{\frac{b k x}{6 a}}}$

and boundary conditions

$u(0, t)=\frac{k\left(-1+c_{1}\right)}{1+c_{1}+c_{2} e^{\frac{b^{2} k^{2} t}{12 a}}} \quad, \quad(1, t)=\frac{k\left(-1+c_{1} e^{\frac{b k}{3 a}}\right)}{1+c_{1} e^{\frac{b k}{3 a}}+c_{2} e^{\frac{b^{2} k^{2} t}{12 a}+\frac{b k}{6 a}}}$

Where $\mathrm{a} \neq 0, \mathrm{~b}$ and $\mathrm{k}$ are arbitrary constants. In this example, $A(u)=a, B(u)=b u$, and

$C(u)=\frac{b^{2}}{9 a} u(u-k)(u+k)$

The exact solutions of this equation have been derived by Andrei D. Polyanin and Valentin F. Zaitsev. [16]

$u(x, t)=\frac{k\left[-1+c_{1} e^{\frac{b k x}{3 a}}\right]}{1+c_{1} e^{\frac{b k x}{3 a}}+c_{2} e^{\frac{b^{2} k^{2} t}{12 a}+\frac{b k x}{6 a}}}$

where $c_{1}$ and $c_{2}$ are arbitrary constants.

Apply the HPM, and equating the terms with the identical powers of $\mathrm{p}$ as:

$p^{0}: \frac{\partial v_{0}}{\partial t}=\frac{\partial u_{0}}{\partial t}$

,$v_{o}(x, 0)=u_{o}(x, t)$

$p^{1}: \frac{\partial v_{1}}{\partial t}=-\frac{\partial u_{0}}{\partial t}+a \frac{\partial^{2} v_{0}}{\partial x^{2}}+b v_{o} \frac{\partial v_{0}}{\partial x}+\frac{b^{2}}{9 a}\left(v_{o}^{3}-v_{o} k^{2}\right)$

,$v_{1}(x, 0)=0$

$p^{2}: \frac{\partial v_{2}}{\partial t}=a \frac{\partial^{2} v_{1}}{\partial x^{2}}+b\left(v_{o} \frac{\partial v_{1}}{\partial x}+v_{1} \frac{\partial v_{0}}{\partial x}\right)+\frac{b^{2}}{9 a}\left(3 v_{o}^{2} v_{1}-v_{1} k^{2}\right) \quad, v_{2}(x, 0)=0$

then solving equation (26), and equation (27), we obtained $V_{1}$ and $V_{2}$ as follows:

$v_{1}(x, t)=-\frac{b^{2} e^{\frac{b k x}{6 a}} k^{3} t\left(-1+c_{1} e^{\frac{b k x}{3 a}}\right) c_{2}}{12 a\left(1+c_{1} e^{\frac{b k x}{3 a}}+c_{2} e^{\frac{b k x}{6 a}}\right)^{2}}$ 
Numerical solution of nonlinear diffusion equation with convection term by HPM

$v_{2}(x, t)=-\frac{b^{4} e^{\frac{b k x}{6 a}} k^{5} t^{2}\left(-1+c_{1} e^{\frac{b k x}{3 a}}\right) c_{2}\left(1+c_{1} e^{\frac{b k x}{3 a}}-c_{2} e^{\frac{b k x}{6 a}}\right)}{288 a^{2}\left(1+c_{1} e^{\frac{b k x}{3 a}}+c_{2} e^{\frac{b k x}{6 a}}\right)^{3}}$

Then the approximate solution of second-order is $u_{2}(x, t)=\lim _{p \rightarrow 1}\left(v_{0}+p v_{1}+p^{2} v_{2}\right)=v_{0}+v_{1}+v_{2}$.

The results are given in the following table and figures:

Table 1: The numerical results for the approximate solution obtained by HPM and comparison with the Exact solution for $c_{1}=c_{2}=1, \mathrm{k}=\mathrm{a}=\mathrm{b}=0.2$

\begin{tabular}{|c|c|c|c|c|}
\hline $\begin{array}{c}\text { Space } \\
X\end{array}$ & $\begin{array}{c}\text { Time } \\
\mathbf{t}\end{array}$ & $\begin{array}{c}\text { Exact } \\
\text { Solution }\end{array}$ & $\begin{array}{c}\text { HPM } \\
\text { Solution }\end{array}$ & $\begin{array}{c}\text { Error } \\
\left|u_{\text {Exact }}(x, t)-u_{2}(x, t)\right|\end{array}$ \\
\hline \multirow{6}{*}{0} & 0 & 0 & 0 & 0 \\
\hline & 0.2 & 0 & 0 & 0 \\
\hline & 0.4 & 0 & 0 & 0 \\
\hline & 0.6 & 0 & 0 & 0 \\
\hline & 0.8 & 0 & 0 & 0 \\
\hline & 1 & 0 & 0 & 0 \\
\hline \multirow{6}{*}{0.2} & 0 & 0.000888882304590150 & 0.000888882304590149 & $2.1684 \mathrm{E}-19$ \\
\hline & 0.2 & 0.000888842798417329 & 0.000888842798417290 & $3.9248 \mathrm{E}-17$ \\
\hline & 0.4 & 0.000888803290488899 & 0.000888803290488587 & 3.1236E-16 \\
\hline & 0.6 & 0.000888763780805095 & 0.000888763780804042 & $1.0538 \mathrm{E}-15$ \\
\hline & 0.8 & 0.000888724269366151 & 0.000888724269363653 & $2.4978 \mathrm{E}-15$ \\
\hline & 1 & 0.000888684756172300 & 0.000888684756167422 & 4.8786E-15 \\
\hline \multirow{6}{*}{0.4} & 0 & 0.001777725104909530 & 0.001777725104909530 & $2.1684 \mathrm{E}-19$ \\
\hline & 0.2 & 0.001777646097830930 & 0.001777646097830850 & 7.8063E-17 \\
\hline & 0.4 & 0.001777567087241040 & 0.001777567087240410 & $6.2428 \mathrm{E}-16$ \\
\hline & 0.6 & 0.001777488073140310 & 0.001777488073138210 & $2.1070 \mathrm{E}-15$ \\
\hline & 0.8 & 0.001777409055529230 & 0.001777409055524240 & $4.9945 \mathrm{E}-15$ \\
\hline & 1 & 0.001777330034408260 & 0.001777330034398500 & $9.7554 \mathrm{E}-15$ \\
\hline \multirow{6}{*}{0.6} & 0 & 0.002666488904294910 & 0.002666488904294910 & $4.3368 \mathrm{E}-19$ \\
\hline & 0.2 & 0.002666370406842970 & 0.002666370406842850 & 1.1753E-16 \\
\hline & 0.4 & 0.002666251904123900 & 0.002666251904122960 & $9.3675 \mathrm{E}-16$ \\
\hline & 0.6 & 0.002666133396138390 & 0.002666133396135230 & $3.1602 E-15$ \\
\hline & 0.8 & 0.002666014882887140 & 0.002666014882879650 & 7.4897E-15 \\
\hline & 1 & 0.002665896364370860 & 0.002665896364356240 & $1.4629 \mathrm{E}-14$ \\
\hline \multirow{6}{*}{0.8} & 0 & 0.003555134221294890 & 0.003555134221294890 & $4.3368 \mathrm{E}-19$ \\
\hline & 0.2 & 0.003554976249264190 & 0.003554976249264030 & $1.5656 \mathrm{E}-16$ \\
\hline & 0.4 & 0.003554818270210270 & 0.003554818270209020 & 1.2477E-15 \\
\hline & 0.6 & 0.003554660284134070 & 0.003554660284129860 & 4.2115E-15 \\
\hline & 0.8 & 0.003554502291036530 & 0.003554502291026550 & $9.9829 \mathrm{E}-15$ \\
\hline & 1 & 0.003554344290918590 & 0.003554344290899090 & 1.9499E-14 \\
\hline \multirow{6}{*}{1} & 0 & 0.004443621597267900 & 0.004443621597267900 & 0 \\
\hline & 0.2 & 0.004443424171710220 & 0.004443424171710030 & 1.9429E-16 \\
\hline & 0.4 & 0.004443226737372940 & 0.004443226737371380 & $1.5595 \mathrm{E}-15$ \\
\hline & 0.6 & 0.004443029294257220 & 0.004443029294251960 & $5.2623 \mathrm{E}-15$ \\
\hline & 0.8 & 0.004442831842364240 & 0.004442831842351770 & 1.2473E-14 \\
\hline & 1 & 0.004442634381695170 & 0.004442634381670800 & $2.4362 \mathrm{E}-14$ \\
\hline
\end{tabular}




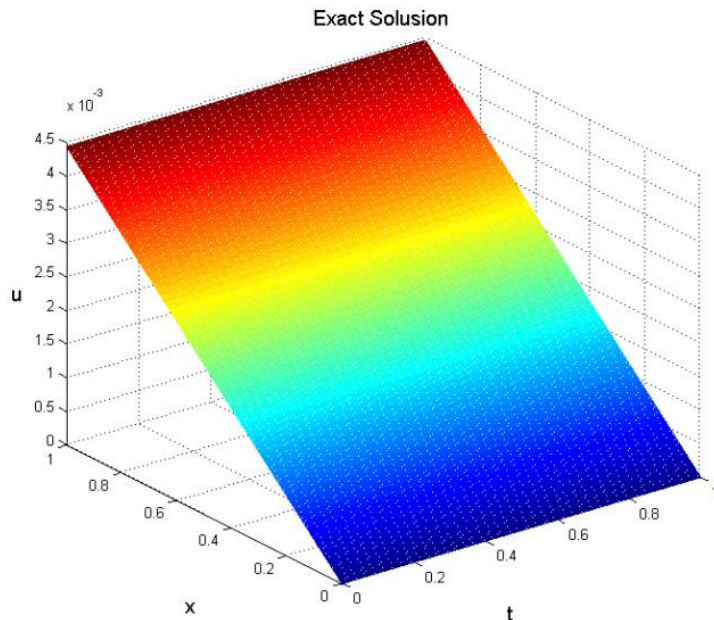

Fig. 1: Exact solution

When $c_{1}=c_{2}=1, \mathrm{k}=\mathrm{a}=\mathrm{b}=0.2$

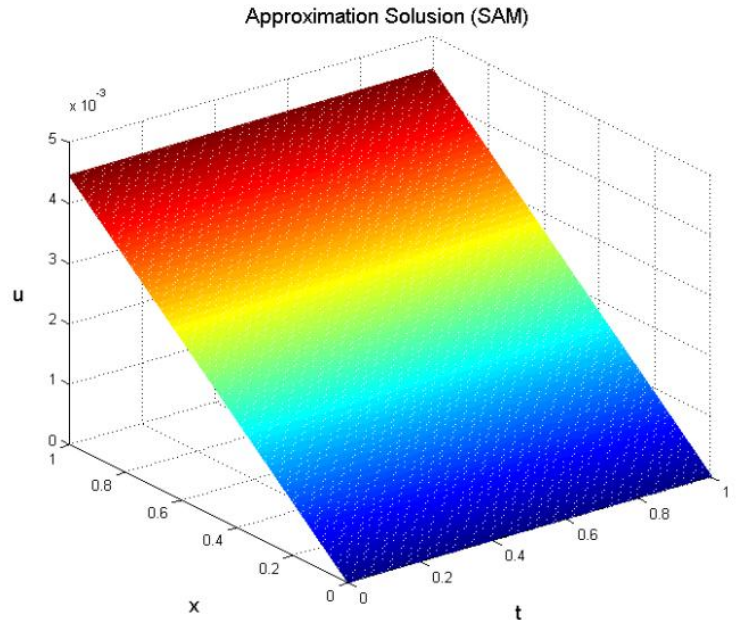

Fig. 2: Approximate solution

When $c_{1}=c_{2}=1, \mathrm{k}=\mathrm{a}=\mathrm{b}=0.2$

\section{CONCLUSION}

In this paper we have applied homotopy perturbation method for approximate solution of nonlinear diffusion equation with convection term and compared with exact solution. We can conclude from the numerical results Fig. (1) and Fig. (2) shows the comparison between the exact solution and the numerical solution obtained by homotopy perturbation method (HPM), For $c_{1}=1, c_{2}=1, k=0.2$, a $=0.2$, and $b=0.2$.It can be seen that the solution obtained by the present method is nearly identical with that given by exact solution. The absolute error $\left|\mathrm{u}_{\text {exact }}(\mathrm{x}, \mathrm{t})-\mathrm{u}_{2}(\mathrm{x}, \mathrm{t})\right|$ of the example be observed in Table (1).Therefore, this method can be seen as efficient method for solving nonlinear diffusion equation with convection term. In our work, we use the Mat lab program to calculate the series obtained from this method.

\section{REFERENCES}

[1] J.H.He, Variational iteration method: a kind of nonlinear analytical technique: some examples, International Journal of Non-Linear Mechanics, 34, 699-708,1999.

[2] He J.H., Homotopy perturbation method: a new nonlinear analytical technique, Applied Mathematics and Computation, 135, 73-79, 2003.

[3] J.H. He, Homotopy perturbation technique, Computer Methods in Applied Mechanics and Engineering, 178, 257-262,1999.

[4] J.H.He, A coupling method of homotopy technique and perturbation technique for nonlinear problems, International Journal of Non-Linear Mechanics, 35, 37-43, 2000

[5] J.H.He,Comparison of homotopy perturbation method and homotopy analysis method,applied Mathematics and Computation, 156, 527539,2004 .

[6] J.H. He, A review on some new recently developed nonlinear analytical techniques, International Journal of Nonlinear Sciences and Numerical Simulation, 1, 51-70,2000.

[7] S.Abbasbandy, Modified homotopy perturbation method for nonlinear equations and comparison with adomian decomposition method,Applied Mathematics and Computation, 172, 431-438,2006.

[8] M.Ghasemi, M.Tavassoli Kajani, A.Davari, Numerical solution of two dimensional nonlinear differential equation by homotopy perturbation method, Applied Mathematics and Computation, 189, 341-345,2007.

[9] M.Ghasemi, M.Tavassoli Kajani, Application of He's homotopy perturbation method for linear and nonlinear heat equations, Mathematic Scientific Journal, 1, 17-27, 2008.

[10] M.Ghasemi,M.Tavassoli Kajani,E.Babolian,Numerical solutions of the nonlinear Volterra-Fredholm integral equations by using Homotopy perturbation method,Applied Mathematics and Computation, 188, 446-449,2007.

[11] M.Ghasemi,M.Tavassoli Kajani, E.Babolian,Application of He's homotopy perturbation method to nonlinear integro-differential equations,Applied Mathematics and Computation, 188, 538-548.2007.

[12] J.H. He,"The homotopy perturbation method for nonlinear oscillators with discontinuities",Applied Mathematics and Computation, 151, 287-292.2004.

[13] R. Cherniha and M. Servo, Lie and non-lie symmetries of nonlinear diffusion equations with convection term, Symmetry in Nonlinear Mathematical Physics, 444-449,2,1997.

[14] He J.H.,'Bookkeeping parameter in perturbation methods," International Journal of Nonlinear Sciences and Numerical Simulation, 2, 257264,2001

[15] A.H Nayfeh, John Wiley, Problems in Perturbation, (New York, 1985)

[16] V.F Zaitsev and A.D. Polyanim,Handbook of Nonlinear Partial Differential Equations,(Chapman and Hall/CRC Press, Boca Raton, 2004). 